\title{
The Infostations Challenge: Balancing Cost and Ubiquity in Delivering Wireless Data
}

\author{
Richard H. Frenkiel, B. R. Badrinath, Joan Borràs and Roy D. Yates \\ Wireless Information Network Laboratory (WINLAB) \\ Rutgers, The State University of New Jersey \\ 73 Brett Road, Piscataway, NJ 08854-8060
}

Corresponding author: Roy D. Yates, ryates@winlab.rutgers.edu

\begin{abstract}
Infostations provide a new way to look at the problem of providing high data rate wireless access. By allowing delayed message delivery, we can lift the constraint on ubiquitous coverage inherited from voice cellular systems. The reduction of coverage results in significant capacity gains, showing the possibility for low cost broadband wireless data services. We give an overview of ongoing research on the Infostation concept.
\end{abstract}

Keywords - Infostations, High data rate, Wireless data, Discontinuous coverage

\section{INTRODUCTION}

In the first 15 years of the cellular revolution, voice services have become commonplace and ubiquitous, and the attention of researchers has shifted to wireless information. Here we find a rather dramatic contrast between expectations and results. It seems clear that information services have an almost unlimited potential in a wireless environment. Many of today's "fixed" applications will continue to make sense in a mobile environment, and a variety of new services are certain to evolve for people on the move. The total number of bits required for voice is limited, even for the most talkative among us, but "information bits" are limited only by imagination and time. Even for the communication we think of as "voice", a significant fraction is already in the form of messages that could be transferred as information files.

Against this backdrop, it is curious that wireless information services have been slow to develop, and have repeatedly disappointed both prophets and financiers. The most common explanation offered is that current radio channels are designed for voice and are too slow for information-hungry applications. As a result, planning for third generation (3G) wireless systems is heavily oriented toward much higher bit-rates.

Researchers at WINLAB at Rutgers University believe that bandwidth and bit rate, while important, are only part of the problem. They have identified the additional factors of cost, battery limitations and the user interface as co-conspirators, and have proposed a radical new architecture called Infostations [1] to address these factors. In this architecture, small and separated islands of coverage provide low-cost, low-power access to information services in a mobile environment. This will satisfy the needs of many data-intensive applications and result in a new class of drive-by services.

The issue of cost-per-bit is particularly important. It is created by the "free bits" routinely provided by the Internet. Coupled with

J. Borràs is also with the Applied Math and Telematics Department, Technical University of Catalonia (UPC), Barcelona, Spain. increasing delivery rates, this strange new economic model has trained a generation of users to download huge volumes of information in search of a single golden nugget, while placing few constraints on the designers of web sites or services. Copper and fiber have yielded increasing bit-rates without increasing costs, creating a sort of economic miracle in which issues of file size are of minimal importance.

In wireless systems, the situation is rather different. Despite continuing advances in technology, reductions in the cost of a minute of conversation have been modest. The most significant cost reductions have been the result of improved voice processing, a reduction in the number of bits rather than the cost of a bit. If a megabitper-second is delivered to the user by a wide-band third-generation wireless system, it remains the equivalent of about 100 simultaneous voice conversations - we get that huge file faster, but not necessarily cheaper.

Thus we are faced with a wired Internet that encourages everincreasing volumes of information, and a wireless technology whose cost per bit is relatively high, and not decreasing quickly. In the face of this economic difference between fixed and mobile information services, it is possible to proceed down different paths toward a solution. One might design separate mobile information applications that require fewer bits. This, however, will greatly reduce the number of applications available to the mobile user, and require the user to learn different services for the wired and mobile different environments. One might process mobile information to reduce the number of bits before transmission. Voice and image processing provide many examples of success on this path. Finally, one might design systems in ways that reduce the cost per bit. It is on this path that we believe the most dramatic results are possible. Infostations are an example of this approach.

\section{The Problem of Coverage}

The root problem is that existing systems (and planned 3G systems) are designed primarily for voice, and must offer anytime, anywhere service. Users wish to receive and place calls at any location and without delay, to move while talking without interrupting their conversations. This requires ubiquitous coverage, which in turn requires significant infrastructure. A modern cellular system is installed with hundreds of base stations, at a cost of hundreds of millions of dollars, in order that a communication link is always available.

Moreover, for a voice system we need to transmit some particular 


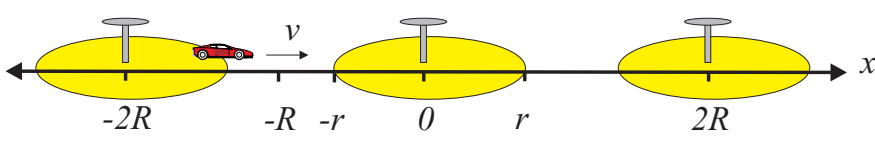

Fig. 1. Highway Infostation model

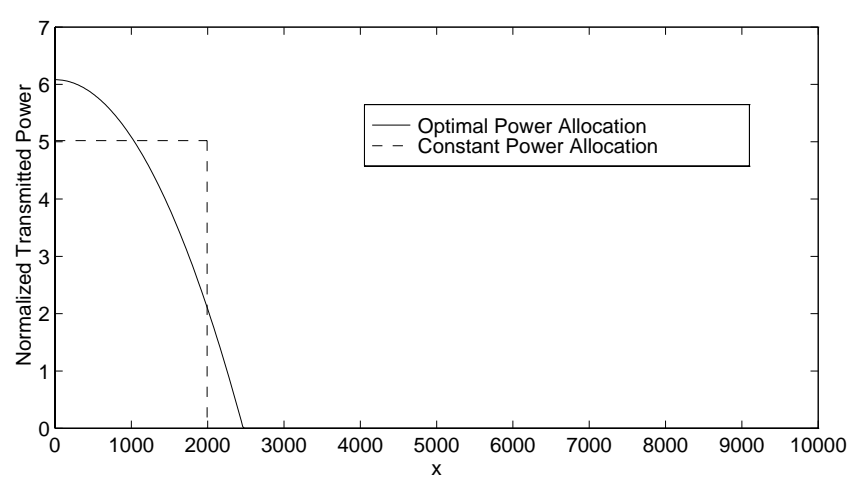

Fig. 2. Example of optimal and constant power allocations $(R=10000)$, normalized by average power

bit-rate, rather than a particular number of bits. Because propagation is highly variable in real-world environments [3], such systems encounter a wide range of channel quality, and must provide an acceptable voice channel with high probability. Thus the channel is designed to be acceptable at the limits of coverage, and the better quality that is available most of the time (and which could offer a higher bit rate) is typically wasted.

In a system designed for data, sizeable delay is often acceptable. This opens the possibility that ubiquity of coverage may not be needed, as long as we achieve a sufficiency of coverage to meet users' needs. In contrast to anytime, anywhere service, we refer to this as manytime, manywhere service. If access to information is provided in the right types of location (toll booths, street corners, building entrances, train stations, airport waiting areas) one may perceive the service as ubiquitous, even where a small fraction of the geographic area is actually covered. The vast array of contiguous cells which is needed in voice systems to provide ubiquitous coverage is reduced to a relatively small number, with a dramatic reduction in infrastructure. This is the key to the low cost of the Infostations architecture.

These ideas can be formalized by examining Shannon capacity as a function of geographic position. Consider the highway Infostation model of Figure 1 in which Infostations are regularly spaced every $2 R$ meters and a mobile moves at a constant speed $v$. When we consider the link to the closest Infostation, we observe a timevarying channel. When the system is subject to an average power constraint, we can apply the methodology developed in [4] to find the capacity of time-varying channels. In the highway Infostation system, the result is an optimal transmit power profile as a function of user position $x$. This optimal power profile is a water-filling policy and is shown in Figure 2 (where $x=0$ is the Infostation location, $x=R=10000$ is halfway the next Infostation) $[5,13]$. The result may seem somewhat surprising in that more power is allocated when the terminal is close to the Infostation rather than far away. The rationale behind this fact is that the power is saved for the epochs when the channel conditions are favorable. Beyond a certain threshold distance, the transmitter is simply shut off. Thus, we see that optimizing channel capacity is consistent with the Infostations concept of restricted coverage areas. Further, if we just use a constant power allocation but with reduced coverage (see Figure 2 ), the loss in average data rate with respect to capacity is marginal $[5,13]$. Average capacity is not so sensitive to the shape of the power control used as it is to saving power for the epochs when the channel is good, that is, to the existence of a cutoff distance for communication.

In what follows, we focus on the downlink channel since it is envisioned that data flow will be highly asymmetrical, with 50/1 ratios or more favoring the downlink $[16,17]$. Downlink interference calculations are somewhat simpler than those for the uplink since the sources of downlink interference are stationary transmitters. Nonetheless, essentially the same arguments for an Infostations architecture also apply to uplink communications. Moreoever, efficient utilization of the limited battery power of the mobile terminal is an added incentive to employ an Infostations system for the uplink.

\section{VARIABLE RATES}

The Infostations channel is not tied to the constant bit-rate of a voice system. As the user moves through the coverage area, the bit rate can be adjusted to reflect changing channel quality. We can do this adaptation through several means: variable bandwidth, variable modulation or adaptive coding. Optimizing throughput [5] and minimizing terminal complexity $[2,6]$ become key areas of study.

For instance, consider varying the modulation in the highway model of Figure 1 as the mobile terminal moves at a constant speed, $v$ past the Infostation at $x=0$. The terminal approaches the Infostation reaching a minimum distance $d_{\text {min }}$ from the Infostation antenna before moving away from the Infostation. As an example, we choose three DPSK modulations, namely DBPSK, DQPSK and 8-DPSK, in a Rayleigh fading channel with propagation exponent of $n=4$ and a required BER of $10^{-3}$. The corresponding required SNR values are 27, 32, and $37 \mathrm{~dB}$, respectively [15]. Figure 3 shows the contour of these required SNR levels, as a function of normalized location $x / d_{\min }$ and $\alpha$, the SNR at location $x=0$. The value $\alpha$ gives a measure of the overall quality of the communication channel. When $\alpha$ increases, we are able to use higher level modulations.

To see the impact of varying the modulation, we can calculate the number of bits $I$ that can be delivered when using a single modulation against using the best at any moment. Note that $I$ equals the time within coverage times the data rate used. We plot a normalized $I$ in Figure 4 as a function of $\alpha$, for the three modulations in the example as a single rate, and also when using the best modulation at every location. In the latter case, $I$ is a summation of contributions from each of the modulations and their corresponding coverage areas. The normalization divides $I$ by the lowest data rate used and the time it takes to travel $d_{\min }$. For instance, if DBPSK yields a data rate of $1 \mathrm{Mbps}$, the minimum distance is $d_{\min }=30 \mathrm{~m}$ and the mobile speed is $v=15 \mathrm{~m} / \mathrm{s}$, Figure 4 corresponds to $I$ ranging from 0 to 24 Mbits. Depending on channel quality, reflected by $\alpha$, there will be a best choice of single modulation. When $\alpha$ is small, higher level modulations will either not be usable or the location range will be too small. As quality improves, higher level modulations become the best choice. With variable modulation, we can always transmit 


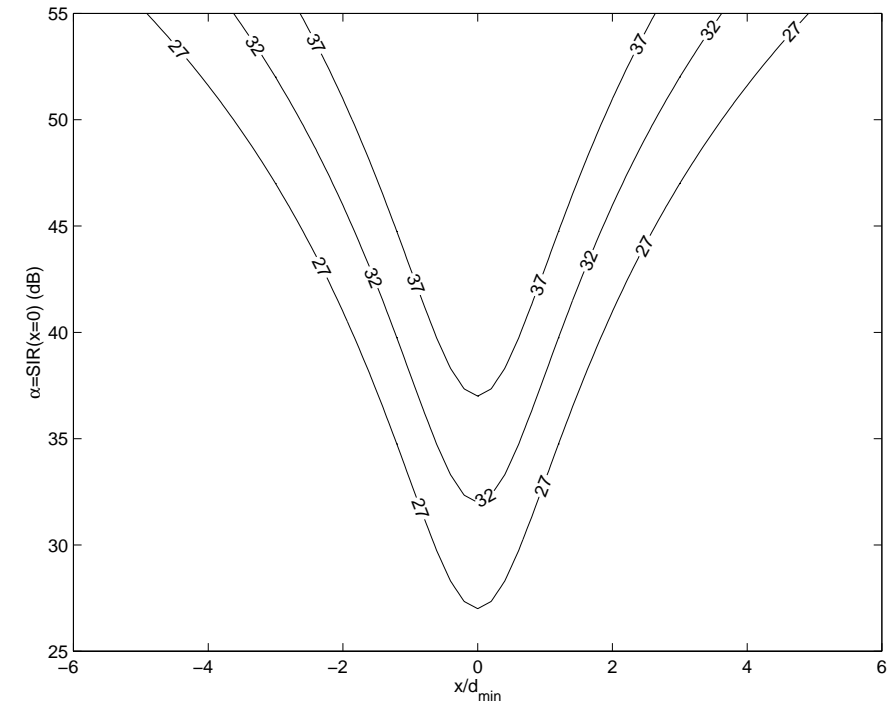

Fig. 3. SNR contour as a function of normalized location $x / d_{\min }$ and $\alpha$, the SNR at $x=0$

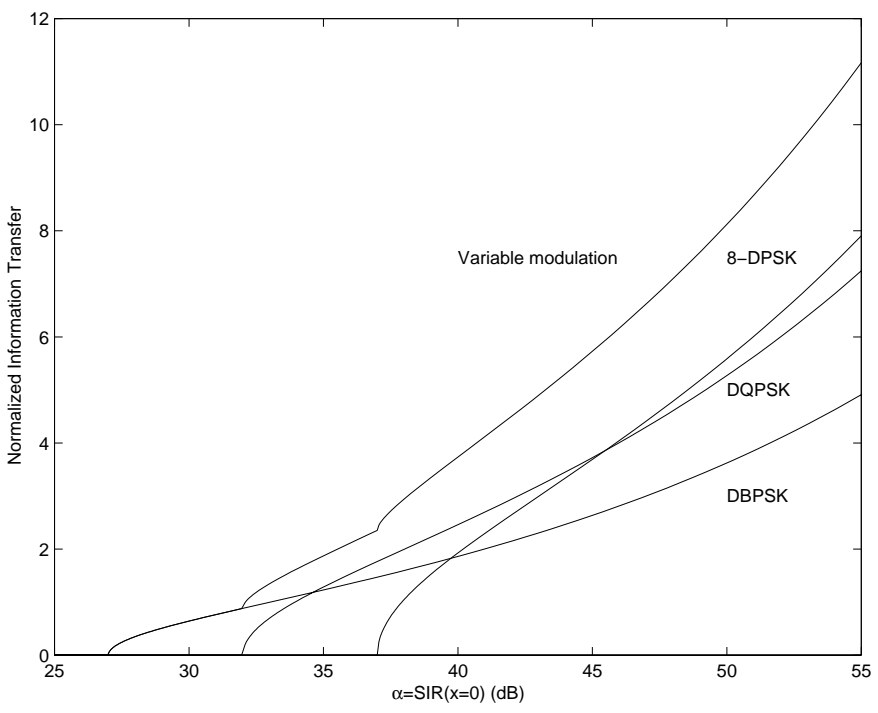

Fig. 4. Normalized information transfer as a function of the $\operatorname{SNR} \alpha$ at the minimum distance

at the highest possible rate. From Figure 4,we observe that the improvement over using a single modulation can be substantial, even given the rather small zones where we would use lower level modulations. Of course, these gains will be reduced somewhat since changing modulations may require resynchronization.

Similar to the constraint on coverage, the anytime, anywhere nature of voice service requires simultaneous access by many users at each base station. Service is offered simultaneously to all who request it, regardless of their location. A user on the boundary of coverage does not wait for the better channel that may eventually become available as he passes through the core of the coverage area. But in an information system with several rates, we can choose to serve the users sequentially, giving the capacity at any time to the user who is able to download information most quickly. This fact alone will increase the capacity of the system.

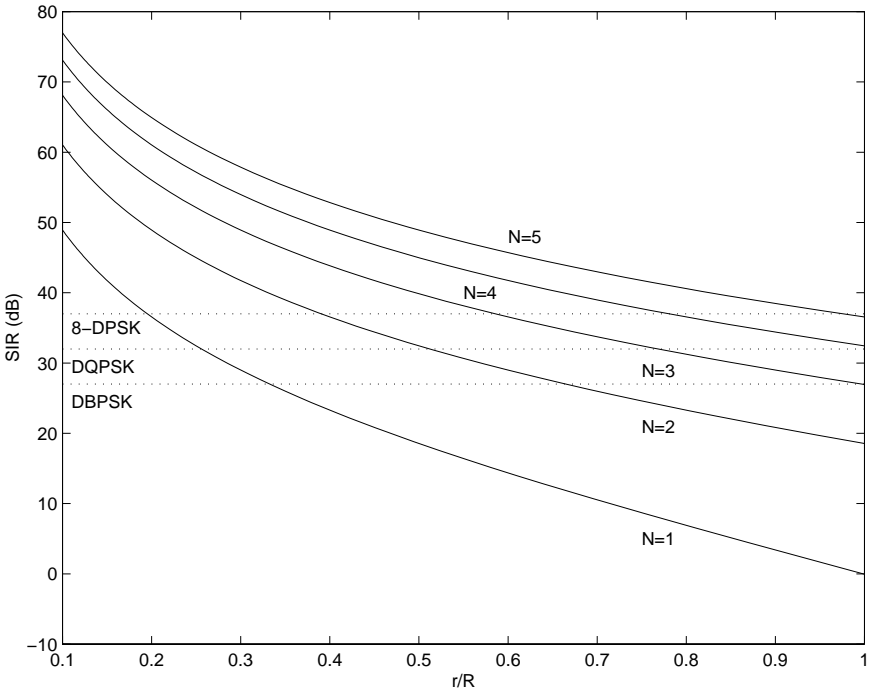

Fig. 5. SIR as a function of $r / R$ for a one-dimensional system

\section{One-Dimensional Coverage: Highway INFOSTATIONS}

These advantages of the Infostations architecture can be seen most easily in the linear, highway model. In a modern, conventional microcell architecture, a highway is covered by a series of base stations creating small but contiguous coverage areas. If we eliminate most of these base stations for an information system, the only result is a small delay in receiving the needed information. Moreover, if we have two vehicles in a coverage area at the same time, we do not need to serve them simultaneously. If one vehicle is near the center of the coverage area (and experiencing excellent coverage), while another is just entering the coverage area (and experiencing marginal coverage), we can provide the full capacity of the base station to the better positioned vehicle while delaying service to the other. This allows a maximum throughput at all times, and further reduces the cost-per-bit. The WINMAC protocol under design at WINLAB provides algorithms for selecting bit rate to match channel conditions. It also addresses issues of error detection/correction and fairness $[7,8]$.

In the highway model with an Infostation every $2 R$ meters, we can view the system as formed by one-dimensional cells of radius $R$. The coverage, though, is not ubiquitous, so that we can speak of a coverage radius $r$, smaller than the cell radius. In spite of the distance between the Infostations, they might still interfere each other. But unlike a voice system, now the worst-case location in terms of SIR is a design choice. Depending on the modulation and the frequency-reuse cluster size, we will have a certain maximum coverage radius $r$. This can be seen in Figure 5, where we plot the worst-case SIR for several cluster sizes as solid lines and the required SIR for several modulations as dashed lines, as a function of $r / R$. The intersections of these lines show possible operating points (in voice systems we would restrict to $r / R=1$ ) [12]. The possibility of a reduced coverage means a dramatic increase in capacity with respect to usual cellular systems. The reason is twofold: reduced coverage allows smaller frequency-reuse cluster size and higher level modulations. The first effect leaves more bandwidth available per Infostation whereas the second improves 


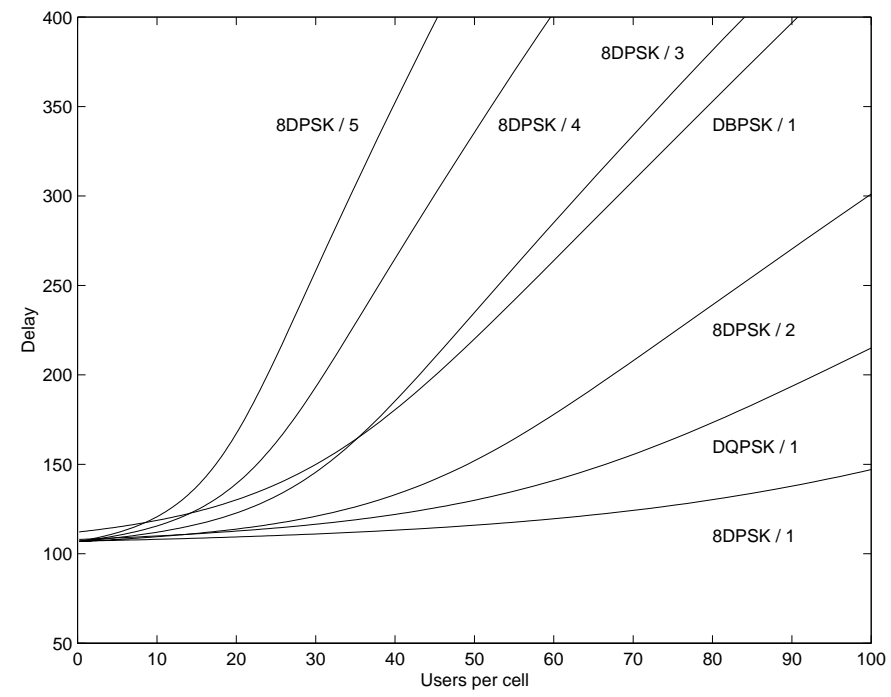

Fig. 6. Average delay as a function of load for highway Infostation

the efficiency we get per unit bandwidth. For example, suppose we compare to DBPSK with cluster size $N=3$ (with full coverage) to 8 -DPSK combined with cluster size $N=1$ (coverage to cell ratio of $19 \%$ ). First, we observe that 8-DPSK transmits three bits per symbol instead of one bit per symbol. Second, the $N=1$ cluster size allows the 8-DPSK system to increase the symbol rate by a factor of 3 over the system with $N=3$ cluster size. Taken together, the 8-DPSK system offers a date rate 9 times higher than the DBPSK system.

However, capacity provides only a partial description of the service characteristics of an Infostations system. We must also examine how delay is affected. In [11, 12], the Infostation was modeled as a queue with reneging, that is, a queue where customers may leave the system before getting service. Using this model, we can calculate the reneging probability, or probability that the mobile leaves coverage before downloading. With this probability and the average time between Infostation visits, we can find the average delay experienced in delivering a message. For the onedimensional highway system, a mobile moving with constant velocity will visit every Infostation, no matter how small the Infostation coverage area. In this case, the time between Infostation visits is essentially independent of the coverage area and delay will be driven by the reneging probability. Figure 6 shows this average delay for several modulations and cluster sizes (possible operating points) [12]. As we can see, the best case is always the highest level modulation with the smallest cluster size. We can conclude that for a highway system, the smallest coverage is best both in throughput and delay.

\section{Two-Dimensional Coverage}

While the linear highway example is instructive, an Infostations system will typically be dispersed in two dimensions. The user will not be constrained to pass through a particular coverage area at all, much less through its center. Moreover, the user may be moving at high speed (vehicular), at low speed (pedestrian) or stationary (sitting in an airport lounge). This leads to an interesting group of problems, in which the creative selection of base station locations and the prediction of the users' paths determine the seeming ubiquity of

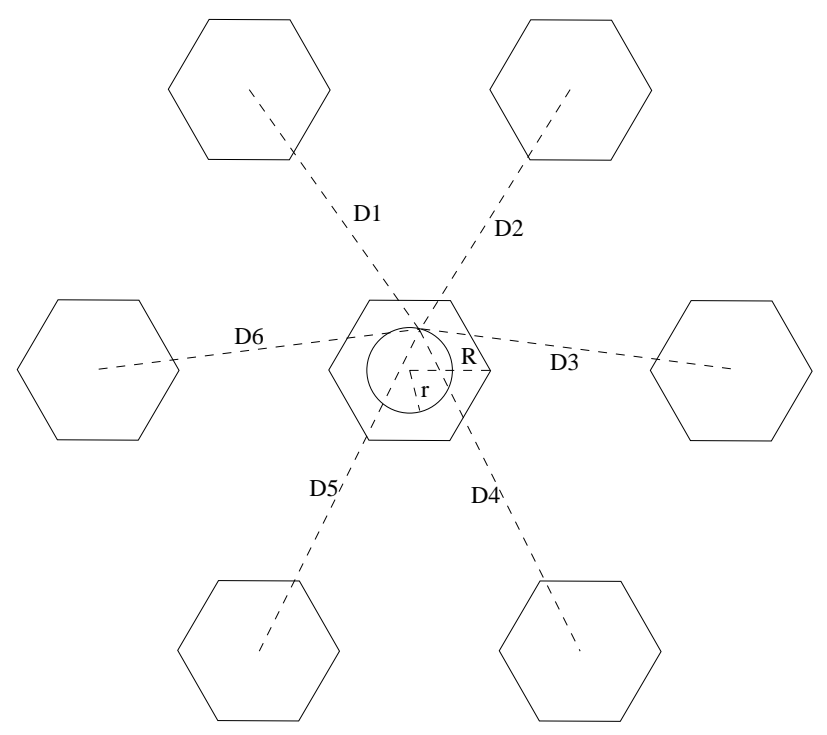

Fig. 7. Two-dimensional Infostation system

coverage and ultimately, the success of the service. The degree to which locations are of a type (e.g., toll booths), and are therefore predictable by the user, may be an important consideration.

To examine coverage issues in a two-dimensional system, we examine Infostations that take advantage of existing cellular infrastructure by using the locations of cellular base stations. Unlike cellular base stations, the Infostations system can sacrifice anytime, anywhere coverage to increase system capacity. To see this, consider Figure 7 in which we have cells with hexagonal geometry and radius $R$, with Infostations in their centers, all of them transmitting at the same power level (we only show cochannel Infostations in Figure 7). The radius $r$ defines the Infostation coverage area, which is generally smaller than the cell area. In the worst-case, the mobile will be at a distance $r$ from the intended Infostation. Figure 8 plots the downlink SIR as a function of $r / R$, for several cluster sizes $N$. The horizontal lines in Figure 8 correspond to the required SIR of several modulations. The result is a set of possible operating points, with different choice of modulation, cluster size and coverage. The highest capacity corresponds to the smallest coverage (as it has the most efficient modulation and universal frequency reuse). For instance, 64QAM and cluster size $N=1$ (radii ratio $25 \%$, area ratio $6 \%$ ) offers a date rate 18 times higher than BPSK with cluster size $N=3$ (full coverage).

The average delay in a two-dimensional layout was also found in [11], for Infostations modeled as a queue with reneging. The mobility model considered is similar to that in [14], with mobiles spread evenly over the area of the cell and traveling in a straight line with direction uniformly distributed between $[0,2 \pi)$. Unlike the highway case, here the time between Infostations visits depends on the ratio of coverage radius $r$ to cell radius $R$. As this ratio is reduced, the more likely it is that a mobile passing through the cell of radius $R$ will miss the Infostation coverage area of radius $r$. This will mean a longer time between Infostation visits. On the other hand, thanks to the increased data rate, a smaller ratio will decrease the reneging probability, which will tend to decrease delay. Hence, there is a tradeoff that makes the two dimensional case somewhat more complex than highway Infostations. Figure 9 shows the average delay for several modulation and cluster sizes. We observe that there is no 


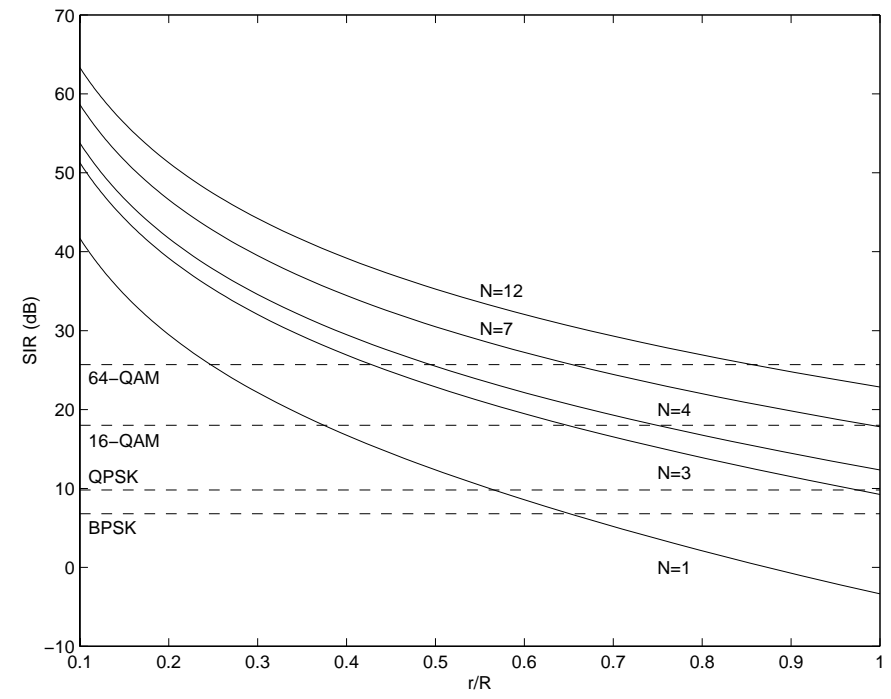

Fig. 8. SIR as a function of $r / R$ for a two-dimensional system

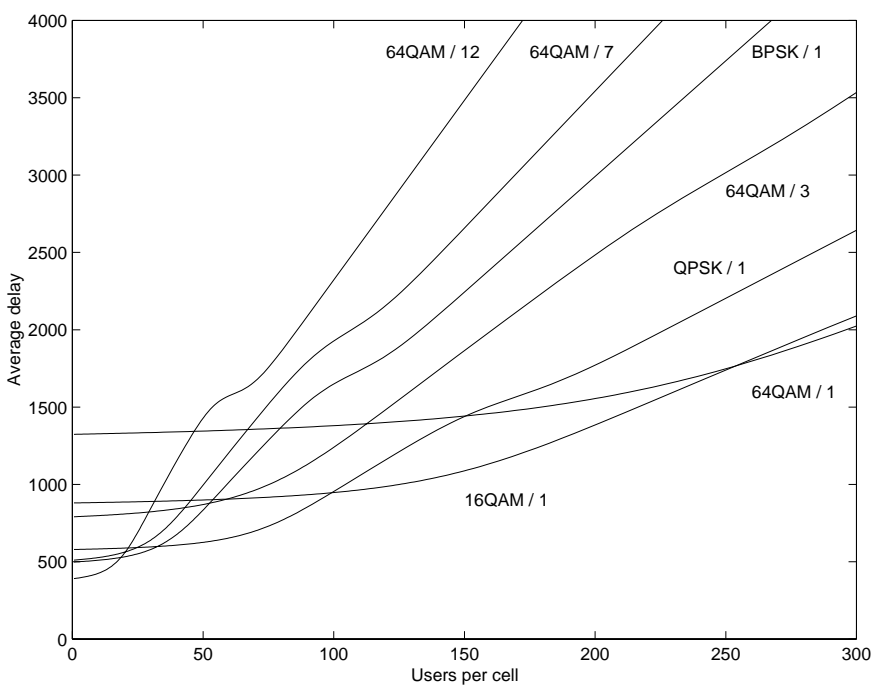

Fig. 9. Average delay as a function of user density in two dimensions

best solution for all cases. In general, for small loads, wider coverage is preferred since the dominant source of delay is the travel time between Infostations. As load increases, the choice moves to higher level modulations and smaller cluster sizes, which with their higher throughput are able to keep delay relatively small. Depending on requirements, we can serve fewer customers with smaller delay, or more customers with a medium delay.

\section{SySTEM INTERWORKING}

No matter how creative and successful is the placement of the Infostations, there remains the possibility that a particular user will not access an Infostation in an acceptable period of time. Moreover, the needed information may be so urgent that immediate and certain delivery is required. This leads to the concept of system choice, in which a user may accept the delay of an Infostation delivery to achieve low cost or reduced battery drain, or may choose immediate service (at higher cost and battery drain) through a ubiquitous cellular, paging or satellite system. By extension, the user may opt for even greater delay and zero cost, by choosing delivery to a fixed terminal at home or office.

This choice of systems leads to an array of interesting problems. How can the service offering be coordinated among systems with such different bandwidth, cost and delay, so that it appears to be a single logical service? To what extent must the content or presentation of the information be changed to match the capabilities of the various systems? What help will the user need to interact successfully with the different systems, and make good choices? It is clear that issues of content encoding and service interface have to be resolved in the context of Infostations [19,20].

Where the user is stationary, or moving slowly, an interactive session between user and system could generally be completed within a single Infostation coverage area. For a fast-moving vehicle, however, or for a pedestrian passing tangentially through the coverage area, the access time may be only a few seconds. How does one discover the Infostation quickly, and how is a request for information provided to the Infostation? Could a ubiquitous low bandwidth network such as paging or CDPD augment the Infostation network and aid in Infostation discovery?

Infostations can serve as a bandwidth booster for a low bandwidth ubiquitous network such as CDPD or two-way paging networks. Users who are normally connected to these networks can use Infostations to get intermittent high data rates. On the other hand, these low bandwidth networks can be used to augment Infostations. For example, a request for a download can be sent via a two-way pager and the actual download can occur at the Infostation. Similarly, once a user passes the Infostation any incomplete transfers can be completed by trickling the remaining bits using the low bandwidth network. At WINLAB, we have implemented a service where a request is sent over a two-way pager and the delivery of the response occurs via the Infostation.

Because the connection may be brief, the session may span several Infostations. Moreover, the path of the user may be uncertain. It may therefore be necessary to cache information at multiple Infostations in order to increase the probability of delivery. Strategies for caching are under study at WINLAB, showing an increase of capacity and decrease of delay [9]. A prefetching algorithm in [21] uses location, route, and speed information to predict future data access, to hide latency from the user. The results suggest that a network design with frequent short range Infostations is better than one with fewer, longer-range stations. Similarly, it may be necessary to reserve capacity at Infostations, in order to assure access during the brief periods of connection [10]. Since users may stay in the coverage area for different lengths of time, and present payloads having different levels of priority, it will be necessary to consider fairness as well as channel quality in assigning the channel. Choices must be made between maximizing throughput and pausing to detect and correct errors $[7,8]$.

The integration of Infostations into a future UMTS was studied in [18], showing the advantages of an Infostations architecture and exploring issues like registration, authentication and billing. They also study the relationship to existing proposals such as GPRS and Mobile IP.

\section{IMPACT ON LAYERED ARCHITECTURE}

At the application layer, an Infostations system will allow a variety of innovative services that would otherwise be impractical from 
a cost standpoint, and will impose a new set of constraints in the delivery of those services. This creates a fertile ground for the invention and design of new services. In addition to personal information services such as email, fax and voice messaging, there is the potential for large-file services (books, magazines, newspapers, music, video) and location-dependent information (advertising, maps or local warnings of hazards). In a military environment, the Infostation can serve as a relay between remote information sources and low-cost, lightweight terminals carried by soldiers.

Finally, the Infostations architecture suggests a new model within the layered structure of communications protocols. Wired communications systems offer stable and predictable performance at the physical layer, with the design of each higher layer built on this fundamental stability and predictability. Wireless systems, in contrast, exhibit dramatic variability at the physical layer, even during a single session. Typically, the designers of wireless systems create the illusion of a stable communication link for higher layers by designing for a minimum channel quality that will be available a high percentage of the time. Such systems do not take advantage of better channel conditions (except through power reduction), and treat poorer channel conditions as an outage.

In an Infostations architecture, the physical layer (and possibly the higher layers) continually adjusts to changing channel conditions, maximizing throughput when conditions allow and pausing during intervals without coverage. While these adjustments seem natural in the case of Infostations, they are a reflection of the true nature of the radio channel and have implications for the efficient design of any radio system.

\section{CONCLUSIONS}

In this paper we have described how wireless data systems are hampered by constraints inherited from voice cellular systems. We have eased these constraints by using a new architecture we call Infostations, which uses small islands of high data rate coverage. Both theory and practice lead to the conclusion that Infostations maximize the capacity of a wireless data system, reducing the cost of the services provided. In one-dimensional systems, we have observed that maximizing capacity is consistent with minimizing delay. In two-dimensional systems, we have identified a tradeoff between maximizing capacity and minimizing message delay.

Although Infostations relax the anytime, anywhere service constraints associated with wireless voice systems, they introduce new issues that derive from the brief, intermittent, and unpredictable nature of the connection between terminals. We have also reviewed the problems associated with the coexistence of Infostations with other systems and the impact on the layered architecture of this new communications paradigm. Resolving these problems is likely to have a significant impact on wireless communication systems.

\section{REFERENCES}

[1] R.H. Frenkiel and T. Imielinski, "Infostations: The joy of "many-time, manywhere' communications", WINLAB Technical Report TR-119, April 1996.

[2] J. Evans, "A Low Cost Asymmetric Radio for Infostations", WINLAB Technical Report TR-130, September 1996.

[3] W.C. Jakes Jr., "Microwave Mobile Communications", Wiley, New York 1974

[4] A.J. Goldsmith, "Design and Performance of High-Speed Communication Systems over Time-Varying Radio Channels", PhD thesis, University of California at Berkeley, 1994
[5] D.J. Goodman, J. Borràs, N.B. Mandayam, and R.D. Yates, "INFOSTATIONS: A New System Model for Data and Messaging Services", in Proceedings of The IEEE Vehicular Technology Conference VTC 97, May 1997.

[6] J.G. Evans, B.R. Badrinath, "Low Sequency W-CDMA Codes Lead to More Economic WLL and Infostation Terminals", (submitted for publication)

[7] G. Wu, C-W. Chu, K. Wine, J. Evans and R. Frenkiel, "WINMAC: a Novel Transmission Protocol for INFOSTATIONS", in Proceedings of The IEEE Vehicular Technology Conference VTC'99, Houston, May 1999.

[8] H. Mao, G. Wu, J. Evans and M. Caggiano, "An Adaptive Radio Link Protocol for Infostations", in Proceedings of The IEEE Vehicular Technology Conference VTC'99, Houston, May 1999.

[9] A.L. Iacono and C. Rose, "Minimizing File Delivery Delay in an Infostations System”, WINLAB Technical Report TR-167, August 1998.

[10] Lee Breslau and Scott Shenker, "Best Effort vs. Reservations: A Comparative Analysis", in Proceedings of the ACM SIGCOMM, 1998.

[11] J. Borràs and R.D. Yates, "Infostation Overlays in Cellular Systems", in Proceedings of WCNC'99, New Orleans, September 1999

[12] J. Borràs and R.D. Yates, "Highway Infostations", in Proceedings of WPMC'99, Amsterdam, September 1999

[13] J. Borràs and R.D. Yates, "Capacity and Power Control: A Simple Model for Infostations", WINLAB Technical Report TR-136, January 1997.

[14] D. Hong and S.S. Rappaport, "Traffic Model and Performance Analysis for Cellular Mobile Radio Telephone Systems with Prioritized and NonPrioritized Handoff Procedures", IEEE Trans. on Vehicular Technology, August 1986, vol. VT-35, no. 3, pp. 77-92. See also: CEAS Technical Report No. 773, June 1, 1999, College of Engineering and Applied Sciences, State University of New York, Stony Brook, NY 11794, USA

[15] J.G. Proakis, Digital Communications, McGraw Hill, 3rd ed., 1995

[16] M. Flament et alii, "An Approach to 4th Generation Wireless Infrastructures - Scenarios and Key Research Issues" in Proceedings of The IEEE Vehicular Technology Conference VTC'99, Houston, May 1999.

[17] T. Sakamoto, K. Tsunoda, E. Kamagata, M. Serizawa, "Network and Protocol Architectures of Hybrid Multimedia Mobile Access Communication System", in Proceedings of The IEEE Vehicular Technology Conference VTC'99, Houston, May 1999.

[18] J. Irvine et al., "Efficient UMTS Data Provision using INFOSTATIONS", in Proceedings of The IEEE Vehicular Technology Conference VTC'98, Phoenix, 1998

[19] H. Bharadvaj, A. Joshi, S. Auephanwiriyakul, "An Active Transcoding Proxy to Support Mobile Web Access", in Proceedings of 17th Symp. on Reliable Distributed Systems, 1998

[20] R. Han et al., "Dynamic Adaptation in an Image Transcoding Proxy for Mobile Web Access", IEEE Personal Communications, vol.5, no.6, December 1998, pp8-17

[21] T.Ye, A. Jacobsen, R.H. Katz, "Mobile Awareness in a Wide Area Wireless Network of Info-stations", in Proceedings of ACM MobiCom'98, Dallas, October 1998 